\title{
An Ultra-Low Power OTA-C Bandpass Filter Design for ECG Application
}

\author{
C. L. Hsiao and R. B. Huang
}

\begin{abstract}
An ultra-low power OTA-C bandpass filter is presented in this paper. An 1-150 $\mathrm{Hz}$ 4-th order OTC-C bandpass filter is design which is suitable for ECG application. A subthreshold region OTA is designed with current cancellation technology to achieve a very small transconductance. The OTA-C filter is design with TSMC $0.18 \mu \mathrm{m}$ CMOS process. The MIM capacitor is used in this design. The supply of the proposed circuit is $1 \mathrm{~V}$. The total power consumption is only $245 \mathrm{nW}$.
\end{abstract}

Index Terms-OTA-C filter, ECG, low power, bioelectronics.

\section{INTRODUCTION}

The development of portable heart monitoring system is not only useful in long-term healthcare, but also demanded in an increasing number of researches in the biomedical file [1]. Filters is continually a critical block of biopotential acquisition systems for enhancing the signal quality [2]. However, for low frequency biomedical applications, realizing filter circuits with large time constant under an acceptable capacitor's value isn't an easy task [3].

Switching-capacitor circuit is a popular technique used in implementing filter circuit. Due to the leakage problem of the advanced process, the sample-hold circuits of the switch-based topologies are not suitable for applications requiring large time constant. Compared with the switching-capacitor filters, continuous-time OTA-C filter has their benefits of lower power consumption and freeing the system from switching noise. The cut-off frequency is proportional to the transconductance $\left(g_{m}\right)$ of the OTA; this allows a low-power implementation for low frequency biopotential signals. In OTA-based circuits, OTA will dominate the performance of the filter circuit, while the ratio of the capacitor to the small transconductance determines the time constant of OTA-C integrators. Thus, an OTA in which the transistors are operated in the subthreshold region is needed to save more power and realize a very low transconductance.

A 4-order Butterworth bandpass is presented in this paper. The bandwidth of this filter is $1-150 \mathrm{~Hz}$. A subthreshold OTA is used to achieve the $1 \mathrm{~Hz}$ cut-off frequency. The transconductance is below $1 \mathrm{nA} / \mathrm{V}$.

Manuscript received October 17, 2012; revised November 24, 2012.

The authors are with the Lunghwa University of Sciense abd Technology, Taoyuan 300, Taiwan, R. O. C (e-mail: CL_Hsiao@mail.lhu.edu.tw, calculus987@hotmail.com).

\section{THE LOW $G_{M}$ OTA DESIGN}

The performance of the OTA is based on the behavior of MOS devices operating in the subthreshold region. The channel current of subthreshold region is:[3]

$$
I_{D}=\left(\frac{W}{L}\right) I_{0} \exp \left(\frac{V_{g s}}{n V_{T}}\right)\left[1-\exp \left(\frac{-V_{d s}}{V_{T}}\right)\right]
$$

where $I_{0}$ is the specific current, $W$ and $L$ are the channel width and length of the MOSFET, $n$ is a weak function of gate voltage and can be considered approximately constant in weak inversion and $V_{T}$ is the thermal voltage.

When the $\left|V_{d s}\right|>4 V_{T}$, the channel current and $V_{d s}$ is almost independence. This is called weak inversion saturation region. The transconductance of the weak inversion saturation cad be expressed as:

$$
g_{m}=\frac{\partial I_{D}}{\partial V_{g s}}=\frac{I_{D}}{n V_{T}}
$$

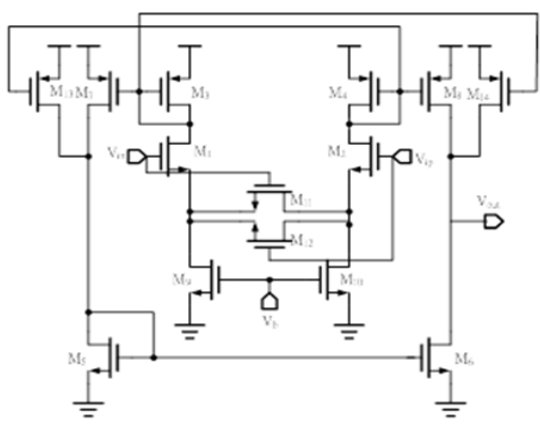

Fig. 1. The proposed low $g_{m}$ OTA design.

The proposed low $g_{m}$ OTA is sown in Fig. $1 . \mathrm{M}_{1} \sim \mathrm{M}_{4}$ are the input stage of the OTA. $\mathrm{M}_{7}, \mathrm{M}_{8}, \mathrm{M}_{13}$ and $\mathrm{M}_{14}$ are current cancellation technology to achieve lower transconductance. If $\mathrm{M}_{3}$ and $\mathrm{M}_{4}, \mathrm{M}_{7}$ and $\mathrm{M}_{8}, \mathrm{M}_{13}$ and $\mathrm{M}_{14}$ are matched respectively, and the $(\mathrm{W} / \mathrm{L})$ of the them is

$$
\left(\frac{W}{L}\right)_{3}:\left(\frac{W}{L}\right)_{7}:\left(\frac{W}{L}\right)_{13}=1: M: N
$$

The output current of the OTA can be written as:

$$
i_{\text {out }}=2(N-M) i_{3}
$$

The overall transconductance of the OTA can be driven as:

$$
G M_{\text {total }}=\frac{\partial i_{\text {out }}}{\partial v}=2(N-M) \frac{\partial i_{3}}{\partial v}=2(N-M) G M_{3}
$$


If $N$ is very close to $M$, then the overall transconductance can be reducing to a very small value.

$M_{11}$ and $M_{12}$ is the source degeneration transistor to improve the linearity performance of the OTA design. The supply voltage of the OTA is only $1 \mathrm{~V} . V_{b}$ is the biasing voltage of the input stage. The total power consumption is only $30 \mathrm{nW}$. The overall transconductance is only $780 \mathrm{pA} / \mathrm{V}$.

\section{The Proposed OTA-C BANDPAss Filter Design}

In order to precisely diagnose the heart disease, the detection circuits must be capable of attenuating the out-of band interference and the noise before analog to digital converter to avoid the aliasing. Ladder type is superior topology for high-order filter because they are inherently insensitive to component variations, especially in their passband. Hence, with the help of filter handbook, a forth-order passive ladder-type bandpass filter is deduced as shown in Fig. 2. The passband of this circuit is $1-150 \mathrm{~Hz}$. The OTA-C bandpass filter is shown in Fig. 3 which is replaced the resistor and inductor in Fig. 2 by OTA-C circuit.

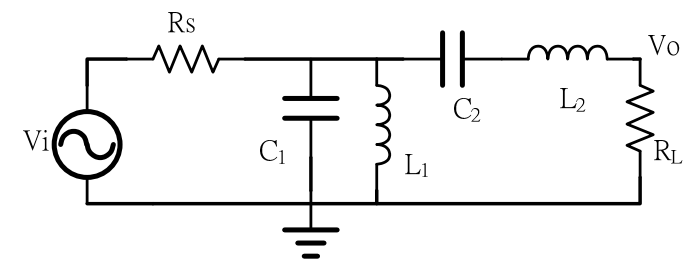

Fig. 2. 4-order bandpass filter topology.

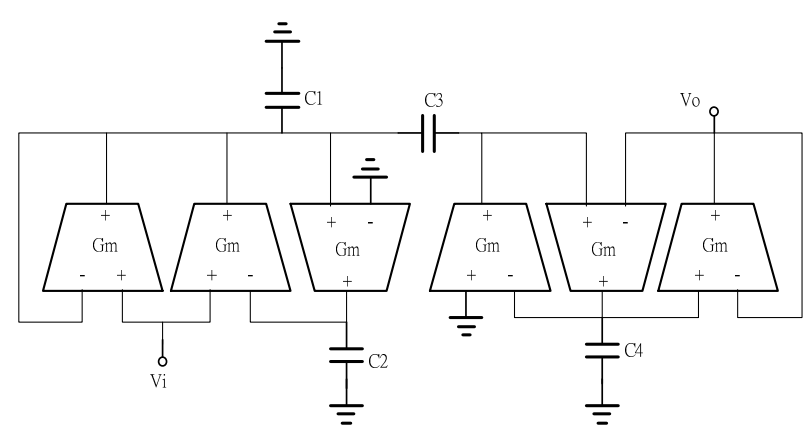

Fig. 3. The OTA-C bandpass filter topology

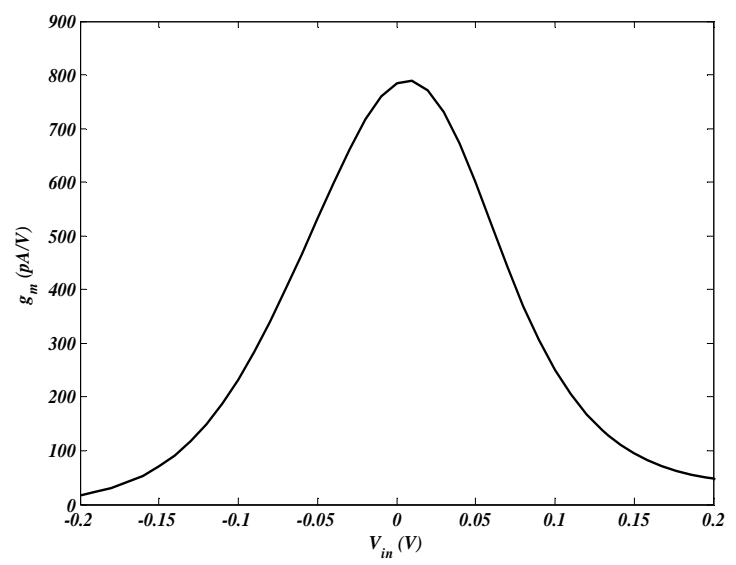

Fig. 4. The transconductance of the OTA.

\section{The Simulation Results}

The proposed OTA-C bandpass filter is designed with TSMC $0.18 \mu \mathrm{m}$ CMOS process. The transconductance of the
OTA is shown in Fig. 4 and the maximum $g_{m}$ of the OTA is $780 \mathrm{pA} / \mathrm{V}$. The frequency response of the OTA-C bandpass filter is shown in Fig. 5 and the passband is about $1-150 \mathrm{~Hz}$. Fig. 6 shows the time response of the OTA-C filter with ECG signal. The supply voltage of the proposed OTA-C bandpass filter is only $1 \mathrm{~V}$. The total power consumption of the filter is $245 \mathrm{nW}$. Fig. 7 is the layout of the proposed OTA-C bandpass filter and the core area is $670 \mu \mathrm{m} \times 280 \mu \mathrm{m}$.

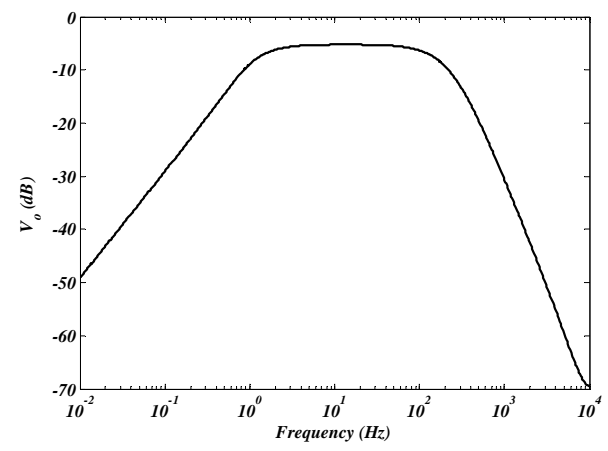

Fig. 5. The frequency response of the proposed OTA-C bandpass filter.
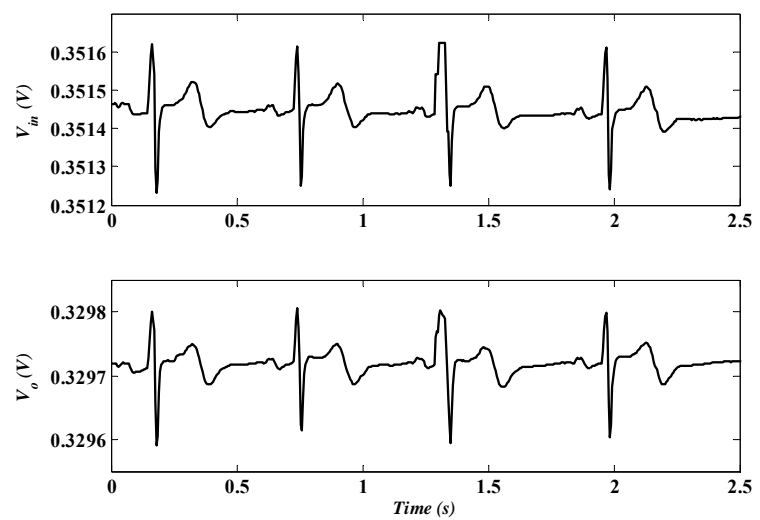

Fig. 6. The time response of the OTA-C filter.

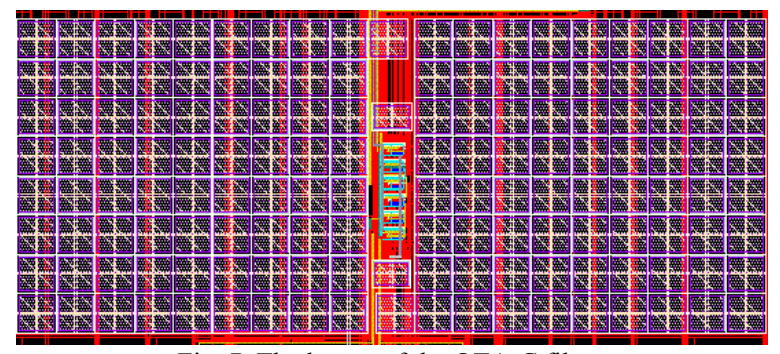

Fig. 7. The layout of the OTA-C filter.

\section{SUMMARY}

An ultra low power OTA-C filter for ECG application is presented in this paper. A subthreshold region OTA is design with current cancellation technology to achieve a very small transconductance. A 4-order Butterworth OTA-C bandpass filter is design for ECG application. The performance summary is shown in Table I. The simulation results show this filter is suitable for ECG application.

\begin{tabular}{cc}
\multicolumn{2}{l}{ TABLE I: SUMMARY OF THE PROPOSED OTA-C FILTER } \\
\hline \hline Supply voltage $(\mathrm{V})$ & 1 \\
\hline Power consumption $(\mathrm{nW})$ & 245 \\
\hline Passband $(\mathrm{Hz})$ & $1-150$ \\
\hline Chip area $\left(\mu \mathrm{m}^{2}\right)$ & $670 \times 280$ \\
\hline \hline
\end{tabular}




\section{ACKNOWLEDGMENT}

The authors would like to thank National Chip Implementation Center (CIC) for technical support.

\section{REFERENCES}

[1] C. H. Tsai, Z. H. Hsieh, and W. C. Fang, “A Low-Power Low-Noise CMOS Analog Frent-End IC for Portable Brain-Heart Monitoring Application," in Proc. of IEEE/NIH Life Science Systems and Applications Workshop, 2011, pp. 43-46.

[2] C. H. Chen, P. I. Mak, T. T. Zhang, M. I. Vai, P. U. Mak, S. H. Pun, F. Wan and R. P. Martins, "A 2.4Hz to $10 \mathrm{kHz}$ Tunable Biopotential Filter using a Novel Capacitor Multiplier," in Proc. of Asia Pacific Conference on Postgraduate Research in Microelectronics and Electronics, 2009, pp. 372-375.

[3] S. Y. Lee and C. J. Cheng, "Systematic Design and Modeling of a OTA-C Filter for Portable ECG Detection," IEEE Trans. on Biomedical Circuits and Systems, vol. 3, no. 1, pp. 53-64, Feb. 2009.

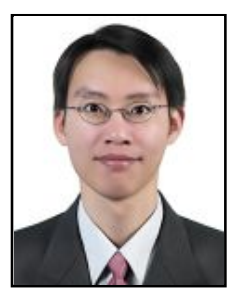

Chih-Lung Hsiao was born in Keelung, Taiwan, R.O.C. in 1976. He received the B.S. degree in Chemical Engineering from Tamkang University, Taipei, Taiwan in 2000 and the M.S. and Ph.D degree in Electrical Engineering from Dong Hwa University, Haulien, Taiwan in 2002 and 2008 respectively. Since 2008, he has been an Assistant Professor in the department of Electrical Engineering, Lunghwa University of Science and Technology, Taoyuan, Taiwan, R.O.C.. His research interests focus on CMOS RF IC design, analog IC design, and bioelectric IC design.

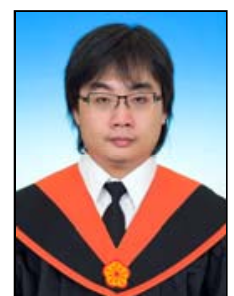

Ren-Bin Huang was born in Chiayi, Taiwan, R.O.C. in 1986. He received the B.S. degree in Electrical Engineering from Lunghwa University of Science and Technology, Taoyuan, Taiwan in 2010. He is currently working toward the M.S. degree in Electrical Engineering at Lunghwa University of Science and Technology, Taoyuan, Taiwan. His research interests focus on analog IC design, and bioelectric IC design. 